\title{
Morbimortalidad femenina en la Puna jujeña: potencialidad de la técnica autopsia verbal
}

\author{
Female morbimortality in Puna jujeña: the potential \\ for verbal autopsy
}

\section{Raquel Irene Drovetta}

Centro de Estudios Avanzados / Universidad Nacional de Córdoba Conicet

Av. Vélez Sarsfield 153

5000 Córdoba - Argentina

raqueldrovetta@cea.unc.edu.ar
DROVETTA, Raquel Irene. Morbimortalidad femenina en la Puna jujeña: potencialidad de la técnica autopsia verbal. História, Ciências, Saúde - Manguinhos, Rio de Janeiro, v.15, supl., jun. 2008, p.257-268.

Desde este espacio proponemos un plan de trabajo que nos permita indagar las particularidades de los procesos de salud-enfermedadatención de las mujeres de las comunidades campesinas de la Puna jujeña, en el norte de Argentina. La etapa en estudio abarca desde inicios de la década del 1990 hasta la actualidad, periodo que se corresponde con la agudización de una crisis socioeconómica que repercutió en todos los ámbitos, incluidos el de la salud. Una forma de dar cuenta de la problemática que afecta a esta población, es el análisis de los procesos de morbimortalidad femenina por causas reproductivas, a fin de determinar la incidencia de los factores socioeconómicos, culturales, étnicos, de género, entre otros, en la instalación de procesos mórbidos y en su desenlace. Para tal fin, utilizamos la autopsia verbal como técnica de recolección de datos, un método cualitativo mediante el cual podemos reconstruir los procesos previos a la muerte según la percepción de los familiares más cercanos de la fallecida para dar cuenta de las particularidades de esta etapa.

Palabras-clave: morbilidad; mortalidad; salud reproductiva; autopsia verbal; Puna (Argentina).

DROVETTA, Raquel Irene. Female morbimortality in Puna jujeña: the potential for verbal autopsy. História, Ciências, Saúde - Manguinhos, Rio de Janeiro, v.15, Suppl., June 2008, p.257-268.

The article proposes a plan for exploring the processes of women's health-illnesscare in peasant communities in Puna jujeña, in northern Argentina. The phase in question runs from the early 1990s to the present time, a period concurrent with a deepening of the socioeconomic crisis that has impacted all spheres, including healthcare. One way of approaching the issues affecting this population is to analyze female morbimortality as related to reproductive concerns and to ascertain the socioeconomic, cultural, ethnic, gender, and other factors involved in morbid processes and their development. Data was collected by verbal autopsy, a qualitative method that allows us to reconstruct processes preceding death, in the eyes of the closest family members. This study is part of doctoral research being undertaken in Agrarian Social Studies at the Universidad Nacional de Córdoba, Argentina.

Keywords: morbidity; mortality; reproductive health; verbal autopsy; Puna (Argentine). 
$\mathrm{N}^{\mathrm{u}}$ uestro objetivo general es determinar la incidencia de factores demográficos, económicos, socioculturales, de género, entre otros, en los procesos de morbimortalidad femenina en la región de la Puna jujeña, desde principios de la década del 90 hasta la actualidad, para conocer en qué forma estos posibilitan o impiden el acceso a diferentes instancias del proceso de atención de la salud y la enfermedad. Esto implica, por una parte, analizar las políticas de salud sexual y reproductiva ofrecidas por los diferentes actores que trabajan en salud en la región, entre ellos el Estado, las ONGs nacionales e internacionales instaladas en la región e inclusos los mismos actores que articulan prácticas médicas de tipo tradicional al interior de las comunidades. Paralelamente, también nos interesa describir las estrategias de acceso a la atención de la salud sexual y reproductiva por parte de las mujeres de la región para conocer de qué forma estas se relacionan con los nombrados proveedores de salud, qué estrategias despliegan, cómo conciben estas prácticas curativas, qué percepciones acerca de la salud y la enfermedad sostienen, etc.

Otro objetivo, este de carácter metodológico, es utilizar la técnica de relevamiento de datos llamada autopsia verbal, para determinar cómo factores ajenos a lo puramente biológico, como las distancias geográficas, la oportunidad de acceso a los servicios de salud, las diferencias de roles según género, etc. inciden en el proceso de morbimortalidad femenina. Si podemos conocer el peso de factores externos, y demostrar que pueden ser revertidos, estaremos en condiciones en el largo plazo de recomendar la planificación de políticas en salud más eficientes y ajustadas a la realidad local.

\section{Contexto y escenario}

Durante la década del 90, en la Argentina, tuvo lugar una agudización en la implementación del modelo neoliberal una de cuyas consecuencias más visibles fue lo que algunos autores denominaron "la retirada del Estado" (Oszlak, mar.-abr. 1999). Este fenómeno consistió en el abandono de los ámbitos de acción en los que históricamente el Estado prestaba atención a sus ciudadanos, con lo cual quedaba definitivamente atrás el modelo que en las décadas del 50 y del 60, incluso hasta entrados los años 70, se experimentó como el Estado de Bienestar. Diversos autores se han ocupado de describir lo acontecido a partir de los años 90 en Argentina permitiéndonos conocer las causas y las consecuencias de las políticas neoliberales implementadas a nivel nacional (Lo Vuolo, Barbeito, 1998; Lo Vuolo et al., 1999; Beccaria, López , 1996; Beccaria, Carciofi, 1996; Lozano, Felletti, 1992; Minujin, 1992).

En particular, los resultados de estas políticas en el ámbito de la salud pública se reflejaron en reducciones presupuestarias, inestabilidad laboral de los prestadores de salud y la carencia de insumos para la tarea cotidiana. Cada uno de estos condicionantes repercutió directamente sobre la salud de la población, revelando la imposibilidad de las instituciones estatales de responder a las demandas sociales con una prestación de calidad. Como contraparte, la sociedad civil reacciona constituyendo innumerables organizaciones del tercer sector, muchas de las cuales sostendrán sus proyectos sociales a través de financiamiento del exterior. Varias de ellas se asentaron en la Puna, suministrando planificación y atención en salud a las poblaciones rurales, en un intento por sustituir la acción del Estado (Roitter, 2004). 
La Puna jujeña es una altiplanicie árida, ubicada a 4.000 metros sobre el nivel del mar, donde las hostiles condiciones climáticas y geográficas hicieron difícil la habitabilidad. Históricamente los pobladores fueron aborígenes de la etnia colla que durante generaciones se dedicaron a la siembra y cría de ganado para autoconsumo. Sin embargo, a partir de la década del 70, cuando se instalan en la zona grandes emprendimientos mineros, muchos jefes de familia comienzan a vender su fuerza de trabajo a cambio de un salario en desmedro del mantenimiento de la unidad doméstica, que ya no puede garantizar la reproducción familiar. Esta población, ya asalariada, recibirá un duro golpe cuando en los 90 la crisis económica y el cierre o las privatizaciones de las empresas lleve el desempleo a cifras record.

Debido a la gran extensión de la región, las poblaciones campesinas están ubicadas a grandes distancias unas de otras. Esto dificulta las comunicaciones, más aún cuando esta zona siempre fue marginada de los procesos de desarrollo nacional no siendo objeto de obras públicas y de infraestructura (Benedetti, ago. 2002).

Varios son los factores que a nuestro entender se conjugan para generar una particular realidad sanitaria en la Puna, entre los que se encuentran la insuficiencia de recursos humanos y materiales. Sin embargo, una de nuestras hipótesis más fuertes es que la ausencia de políticas de salud que conciban el factor cultural de la población aborigen a la que van destinadas, es uno de los factores que más se vincula con los procesos de morbimortalidad femenina.

Entre los indicadores que revelan la gravedad de la situación sanitaria en la zona, podemos señalar el aumento de la cantidad de personas sin cobertura médica (Datos Indec 1991 y 2001; Encuesta Permanente de Hogares), el crecimiento en los índices de mortalidad materna y desnutrición infantil (Romero, Ramos, 2004), así como la detección de una significativa cantidad de casos de mujeres con cáncer cervicouterino (Gronda et al., 2002), información que revelaría la falta de acceso a una adecuada atención en salud sexual y reproductiva de las mujeres y así como una estructura sanitaria deficitaria. .

El perfil epidemiológico de la salud reproductiva en la Argentina nos muestra diferencias según el nivel socioeconómico y de jurisdicción, afectando de manera más pronunciada a las mujeres más jóvenes, las más pobres y a las mujeres con menor nivel de educación, en especial a las que residen en zonas rurales y son indígenas (Figueroa, Stern, 2001).

Frente a este escenario, nos surgen interrogantes acerca de cuál es la profundidad del impacto de esta problemática sobre los diferentes grupos sociales a los que nos referimos. ¿Qué tipo de estrategias y políticas de acción se generan, tanto a nivel público como privado, para revertir la situación? ¿Cómo impactan los condicionantes económicos y culturales en la salud? Indagando aún más profundamente nos preguntamos ¿qué percepciones acerca de la salud y la enfermedad atraviesan este escenario de situación y qué actores lo componen? ¿Qué modelos de accionar médico sostienen las prácticas sobre la salud femenina? Incluso ¿qué implicancias para la propia salud sexual y reproductiva tiene el ser 'mujer indígena'?

\section{Algunas definiciones teóricas}

La población en estudio presenta altas tasas de pobreza, baja calidad de vida e incluso necesidades básicas insatisfechas (Informe Desarrollo Humano, 1996). En este sentido, la 
situación actual evidencia que las políticas de salud destinadas a mujeres en edad reproductiva no fueron totalmente eficaces y se carece de datos que muestren cambios substanciales en las condiciones de acceso a la salud sexual y reproductiva de las mujeres en la última década. Por el contrario, muchas mujeres continúan viviendo situaciones de alto riesgo

El conjunto de acciones e iniciativas articuladas en torno a la salud sexual y reproductiva, ya sean del orden privado o público, tanto impliquen estrategias individuales o colectivas, se asientan en concepciones particulares sobre lo que implica la salud y la enfermedad, lo que Menéndez (1992) entiende como diferentes 'modelos médicos'. Desde el punto de vista de la oferta en salud, las instituciones estatales y las ONGs que trabajan en la zona planifican sus acciones en función de un "modelo médico hegemónico, caracterizado por un proceso de concentración monopólico en la atención a la salud y por una función cada vez más directa del Estado" (p.110). Este modelo entra en conficto en tanto intenta la exclusión ideológica y jurídica de los otros modelos alternativos (p.98).

Sin embargo, aún cuando esto sucede, al interior de las comunidades persisten las prácticas basadas en el modelo médico tradicional que incluyen el curanderismo, el chamanismo, el uso de hierbas medicinales etc. Según nuestra hipótesis, el acceso a la atención de los padecimientos a través del sistema de salud oficial, está mediada por la selección que hace el enfermo, de acciones 'previas' de atención a su salud. Esto significa la preferencia de 'técnicas de autotratamiento' como primera estrategia y sobre la propia dolencia, 'y del curanderismo' como paso posterior en caso que fracase el autotratamiento, combinando generalmente esta atención con la biomédica (Idoyaga Molina, 2001).

La inexistencia de un trabajo conjunto entre estos modelos de atención, biomédico y tradicional, sin que ello implique la superioridad de uno sobre otro, se debe fundamentalmente a que las prácticas de atención y curación tradicionales fueron estigmatizadas por el modelo médico hegemónico por lo que permanecen activas, pero en un segundo plano, reservadas para ser utilizadas exclusivamente al interior de las comunidades.

Todas estas experiencias están atravesadas por construcciones valorativas, redes de significados construidos socialmente, subjetividades en juego, e inclusive por la conjunción del saber científico y el sentido común. Esto hace que nos hallemos ante un escenario heterogéneo, a partir del cual se desprenden líneas de acción que repercuten en la forma en que la población femenina se relaciona con su salud sexual y reproductiva, dando lugar a lo que entendemos 'son respuestas sociales' con diferentes alcances y resultados. Esto da cuenta de cómo la salud sexual y la reproducción humana no deben ser entendidas simplemente como hechos biológicos, sino como un complejo fenómeno de índole social y cultural, sostenida por significados que son construidos socialmente, plausibles de ser decodificados si nos adentramos en la manera en que los diferentes actores involucrados conciben este hecho. Incluso, debemos tener en cuenta que cada grupo social accede al conocimiento médico y sus prácticas en función de factores tan intrínsecos como su escala de valores, mentalidad, expectativas, redes de significado construidas y status social (Salgado Martínez, 2001, p.124). 


\section{Conceptos en torno a la salud femenina}

Como ya enunciamos, a lo largo de este trabajo centramos nuestro objetivo en estudiar los procesos de morbimortalidad reproductiva, acontecidos entre la población femenina de la Puna. Esta definición encierra una serie de dimensiones que dan cuenta de dos momentos fundamentales: el proceso de enfermedad o morbilidad, y el proceso de mortalidad, Esta división temporal, si bien puede parecer arbitraria, es útil analíticamente dado que nos permite cumplir con el cometido de indagar acerca de las particularidades de ambos momentos.

A través del análisis de los procesos mórbidos, podemos dar cuenta de todas las mujeres que cargan, temporalmente o de por vida, con enfermedades, lesiones o discapacidades asociadas al embarazo, el parto y el puerperio, como "las complicaciones por aborto, las consecuencias agudas y crónicas de las enfermedades de transmisión sexual, las consecuenscias psicológicas y físicas de la sexualidad prematura entre los adolescentes y las consecuencias psicológicas y físicas de la coerción sexual", entre otros (Leslie, RubinKutzman, Goldani, 2001, p.180).

Por otra parte, no menos relevante es la mortalidad por causas reproductivas, aspecto que nos permite analizar la gravedad de la problemática socio sanitaria, sobre todo cuando estamos frente a muertes evitables, como la mortalidad materna y la mortalidad por cáncer cervicouterino. Por ambas causas mueren numerosas mujeres en la región en estudio.

Si bien este concepto comúnmente es reflejado a través de indicadores cuantitativos, como las tasas y los porcentajes, nuestro objetivo es analizar el fenómeno desde el aspecto cualitativo, aspecto que ampliaremos en las consideraciones metodológicas.

Para los fines de este trabajo es indispensable tener como referencia un concepto de salud, para lo cual tomamos una categorización amplia que comprende tres instancias: la salud materna, la salud reproductiva y la salud de las mujeres, términos que están en íntima relación, aunque no son sinónimos.

En este caso, la salud reproductiva, que incluye también a la salud sexual, se ubica en el centro "como un continuo entre la salud materna y la salud de las mujeres" (Leslie, RubinKutzman, Goldani, 2001, p.166). Este es un doble avance que hace que la salud sexual signifique más que el acto de la reproducción en si mismo, siendo que en este enfoque es superador de la salud materna y se incluye en el ámbito de la salud de las mujeres.

Hacemos la observación de que utilizamos la definición de salud reproductiva tal como se aplica a las mujeres, sabiendo que queda fuera de esta óptica el análisis del rol masculino en este fenómeno. Esto constituye una decisión metodológica a fin de centrar la problemática en las mujeres, lo que "actúa como un principio organizador para comprender y atender las necesidades de las mujeres en materia de salud" (Leslie, Rubin-Kutzman, Goldani, 2001, p.166).

Como definición, la salud reproductiva ha dejado de tener la significación que se le asignaba anteriormente, ligada a la "salud obstétrica y ginecológica" (Leslie, Rubin-Kutzman, Goldani, 2001, p.165) y desde los años 90 han prevalecido otras definiciones con un sentido más amplio que la referencia a aspectos puramente clínicos o biomédicos.

Sin embargo, debido a que es un proceso reciente, aún no hay acuerdo cierto sobre la significación de lo que se concibe actualmente por salud sexual y reproductiva. No obstante se 
han logrado algunos consensos, como por ejemplo la necesidad de construir un concepto amplio de salud reproductiva que incorpore los contextos económicos, institucional, político y cultural, superando por esta vía el ámbito restringido de lo biomédico (Salles, Tuirán, 2001, p.93).

En tal sentido y a fin de contribuir a la construcción de esta definición, pensamos que tampoco puede ser excluido ningún aspecto sobre lo que implica la reproducción humana (desde lo ético hasta lo económico), así como no debe prescindirse de nociones que refieran a los derechos reproductivos, los derechos sexuales y la salud sexual (Correa, 2001).

Por su parte, el concepto de salud de las mujeres dirige la atención a las necesidades de las mujeres, incluyendo a aquellas que están fuera de la edad reproductiva, así como al diagnóstico y tratamiento de problemas de salud no reproductiva. La riqueza de esta definición radica en que concibe a la salud de la población femenina como diferente de la masculina, no sólo en los aspectos biológicos, ampliamente conocidos, sino por la diferencia en sus roles, los cuales son determinados social y culturalmente, dando lugar así a comprender la incidencia de las prácticas instituidas socialmente, sobre la salud femenina. (Leslie, RubinKutzman, Goldani, 2001, p.174). En otras palabras, la salud de las mujeres se define como algo que incluye la salud reproductiva, "pero que va más allá de ella" (p.176).

Este concepto nos permite indagar aquellas prácticas socialmente naturalizadas, donde la posición de la mujer es desventajosa en relación a la del hombre, como sucede en las comunidades campesinas en estudio. A partir de su rol de madre-esposa, la mujer es concebida como la 'responsable natural' de la reproducción y el consumo familiar, lo que equivale a tener la responsabilidad de 'estar a cargo de la reproducción de otros'. Estas funciones se sostienen en jerarquías de género que son creadas y mantenidas a través de la interacción con los integrantes del hogar, alimentando relaciones primarias de subordinación-dominio entre los géneros, alojadas en la esfera más íntima del hogar (León, 2003). Los indicadores a tomar en el análisis de la salud de las mujeres están relacionados con los roles asumidos por las mujeres a lo largo de los años y las repercusiones que estos previsiblemente tendrán sobre su salud.

Finalmente, la salud materna, constituye la circunstancia que más convoca a las mujeres a las instituciones de salud, quienes la tienen como destinataria y beneficiaria - indirecta - de los programas de salud materno-infantil y de planificación familiar.

Es preciso analizar que muchos de los procesos mórbidos que acontecen en esta etapa son consecuencias de inadecuadas condiciones de vida que se reflejan en problemáticas como la desnutrición, la anemia y los embarazos poco espaciados. En este sentido, la pobreza y los sesgos de género contribuyen a exacerbar estos factores biológicos de riesgo (Leslie, Rubin-Kutzman, Goldani, 2001, p.182).

\section{Especificaciones metodológicas}

La metodología seleccionada para recabar los datos es de tipo cualitativa, donde destacamos fundamentalmente el uso de dos técnicas que nos conducen a obtener información para construir una historia de vida. Por un lado, la entrevista en profundidad sobre la que no daremos mayores detalles por considerar que se trata de una técnica suficientemente conocida. Esta se aplica a la población en estudio que haya pasado por la 
experiencia de sufrir un proceso de morbilidad reproductiva. En tal caso, lo que se pretende es reconstruir, por medio de la entrevista, los acontecimientos vitales previos a la enfermedad hasta la actualidad, información que constituirá el dato para el posterior análisis.

Dado que nuestro interés abarca también a los fenómenos de la mortalidad, para lo cual no nos son suficientes los datos cuantitativos, pretendemos innovar en la recolección de los mismos aplicando la técnica de la autopsia verbal.

\section{La autopsia verbal: antecedentes}

Como su nombre lo indica, esta técnica implica el relevamiento de datos en forma oral que permitan dar cuenta de una serie de acontecimientos ocurridos previamente a la muerte de una persona en relación con este hecho. Para esto, se entrevista a las personas que se considera que han acompañado los últimos tiempos de la persona fallecida.

Es un procedimiento que proviene del área de la salud y ha sido implementado fundamentalmente como forma de medir la mortalidad acontecida en regiones donde existe subregistro de las causas, de ahí que los estudios de caso realizados provengan en su mayoría de países en vías de desarrollo, donde la estructura sanitaria es deficiente y no permite un registro estadístico adecuado de la mortalidad por causa.

Mediante la aplicación de un cuestionario estructurado, si el encuestador no es personal médico, o bien semiestructurado, en caso de que lo sea, se indaga a los parientes más cercanos de la persona fallecida. Los informantes son seleccionados según se considere que han estado presentes acompañando las últimas instancias de la persona fallecida, circunstancia que le permitió ser testigo del avance y desenlace del proceso de morbimortalidad. Este requerimiento es fundamental debido a que la indagación es en torno a la sintomatología que presentó la persona previamente a la muerte.

Esta técnica ofrece la oportunidad de contrastar los datos recogidos en campo con los que figuran en los registros oficiales. Con los datos obtenidos se procede a verificar que los mismos coincidan con el certificado de defunción elaborado por el médico. En esencia, esta técnica copia la experiencia de completar una historia clínica, donde el entrevistado no es el paciente, sino alguien cercano a éste que pueda describir los padecimientos previos a la muerte (Cárdenas, sept.-dic. 2000, p.668).

Los principales antecedentes en su uso son resultado del intento por determinar el subregistro de la mortalidad materna y distinguir entre las causas asociadas al embarazo, el parto o el puerperio. Las primeras publicaciones que dan cuenta de esto en su mayoría son extranjeras y refieren a experiencias recientes, lo que indica que este método está aún poco extendido.

En Argentina, son precursores los estudios del Cedes que a través de un Estudio Colaborativo Multicéntrico que reveló el subregistro de la mortalidad materna en el interior del país (Romero, Ramos, 2004; Ramos et.al 2004).

Una experiencia fundamental para el caso latinoamericano es la desarrollada por Freyermuth Enciso $(1999,2003,2004)$ en el sur de México, donde utiliza la técnica para determinar sub registro de muertes femeninas por causas asociadas a embarazo, parto o puerperio en la región de Chiapas. Por su parte, Castro et al. (2000) también buscan 
factores asociados a la muerte materna en tres estados mexicanos, mientras en la región de Morelos el trabajo de Campero et al. (2006) busca documentar cómo el subregistro impide ver que la violencia es una causa de mortalidad materna. Fuera del caso latinoamericano, similares usos tuvo por ejemplo en el caso de en Bangladesh, donde se buscó comprobar niveles y causas de muerte materna (Ronsman et al., 1998); en Guinea-Bissau para medir la incidencia de la mortalidad materna en población multiétnica (Hoj, Stensballe, Aaby, 1999); a través de un estudio cualitativo del acceso al cuidado obstétrico en la zona rural de Gambia (Cham, Sundby, Vangen, 2005); como modalidad de medición de la mortalidad materna en los países en desarrollo, comparando sistemas de defunción en hogar e historias de hermanas (Hill et al., Mar. 2006); como un modelo probabilístico para determinar las causas de embarazo terminadas en muerte, (Fottrell et al., 2007); proponiendo el uso simplificado de la autopsia verbal para identificar las causas de muerte en adultos en una población predominantemente rural en Etiopía (Lulu, Berhane, 2005). Otros trabajos buscan proponer el método como una alternativa metodológica que permita registrar hechos referidos a mortalidad, utilizándolo como registro por muestreo de acontecimientos y estadísticas vitales, que permitan mantener actualizado el sistema de registro civil (Setel et al., ago. 2005, 2006) El método tiene también antecedentes relacionados a otras patologías, como la tuberculosis, en cuyo caso se buscó determinar, el diagnóstico tardío de la enfermedad, el abandono del tratamiento y su asociación con alcoholismo (Bustamante-Montes, Bellido-Bárcenas, Riojas-Rodríguez, 1996).

La riqueza de esta técnica, a nuestro entender, radica en que más allá de su uso innovador para determinar causas de muerte específicas es posible utilizarla también para "conocer aspectos relacionados a factores sociales, culturales o de atención a la salud que rodearon el desarrollo del padecimiento" (Cárdenas, sept.-dic. 2000, p.667). La potencialidad del relevamiento de estos aspectos que hacen a la salud de la población radica en que son susceptibles de ser modificados mediante el diseño de programas o políticas específicos (p.674), lo que conduce a canalizar las aspiraciones de este tipo de investigación que busca constituir cambios en la realidad de estas poblaciones en el mediano plazo.

\section{La aplicación de la técnica de autopsia verbal}

Sintéticamente exponemos los principales pasos a seguir en la aplicación de esta técnica en la región en estudio.

En primera instancia indagamos en el hospital cabecera, las historias clínicas y los certificados de defunción de las mujeres afectadas por procesos de morbimortalidad reproductivas y constituimos dos grupos de estudios: uno compuesto por aquellas que atraviesan procesos mórbidos y otro constituido por las mujeres fallecidas por causas relacionadas a enfermedades sexuales y reproductivas.

Una variante importante del método en su uso original, es que en este trabajo no pretendemos cuestionar la causa de muerte definida en el certificado de defunción, sino que esta es tomada como condicionante de pertenencia o no al grupo en estudio. Asumimos el subregistro existente en la estadística, siendo nuestro objetivo en cambio entrevistar a 
las familias de mujeres cuyas muertes hayan sido registradas como muerte materna, para en esos casos indagar factores no biológicos que contribuyeron al deceso.

A continuación, clasificamos los casos en dos grandes grupos a indagar: el de mujeres que padecen o padecieron un proceso mórbido relacionado a aspectos sexuales o reproductivos, y el grupo de mujeres cuyo registro indique como causa de muerte la mortalidad materna, a los fines de constituir la muestra Posteriormente localizamos a las mujeres que enfermaron, para el grupo de las no fallecidas, y a los familiares cercanos, para el grupo de las fallecidas. Esta instancia es la que mayores dificultades presenta, entre otras cosas porque es posible que la familia haya migrado y no se tengan datos actualizados de su paradero. Otra alternativa es que se ubique a la familia de la mujer fallecida, pero no se ubique a la madre, la hermana o, en ultima instancia, a la pareja, quienes actuarían como los informantes claves para reconstruir la historia de vida de la persona fallecida. Otra posibilidad, es que los miembros de la familia se nieguen a hablar del tema, lo que nos lleva a seleccionar un nuevo caso

Tanto la entrevista en profundidad como la autopsia verbal nos permite centrarnos en el análisis de la incidencia de factores socioeconómicos, culturales, institucionales, políticos, de género, étnicos, etc., que influyeron de alguna manera en el proceso de morbimortalidad femenina. Para tal fin, la entrevista gira en torno a los indicadores que construimos de cada una de las nombradas categorías.

\section{La memoria como sesgo}

Uno de los posibles cuestionamientos a la validez de los datos a recolectar, es el tiempo transcurrido desde el hecho hasta la aplicación de la autopsia verbal.

Indagando en los antecedentes, hemos encontrado que el período recomendado para la aplicación de la técnica a los familiares varia enormemente, entre tres meses y diez años (Cárdenas, sept.-dic. 2000, p.672). En el caso de mayor transcurso de tiempo desde el fallecimiento, los investigadores demostraron que los datos volcados a la entrevista correspondían con la sintomatología descripta en la historia clínica y con la causa de defunción registrada originalmente (Hoj, Stensballe, Aaby, 1999). Sin embargo, para los fines de este trabajo, se planificó aplicar la técnica a familiares de una mujer fallecida con una anterioridad de hasta cinco años como máximo. De esta forma, es probable que nos encontremos con casos de mujeres fallecidas a inicios de esta década, pero cuyo proceso mórbido se inició bastante antes, con lo cual podremos ahondar en aspectos relevantes del trabajo, como por ejemplo las políticas de salud de la que fue destinataria durante los años 90 .

\section{Potencialidad de la técnica}

Como explicamos al inicio del trabajo, uno de nuestros objetivos es de orden metodológico y se relaciona con la intención de determinar la potencialidad de esta técnica para recabar información relevante, sobre todo cuando refiere a indicadores plausibles de ser modificados con una política acorde. 
En este sentido, estamos esperanzados en hacer de este un método cada vez más afinado, que permita acceder a información privada que no es relevada en las historias clínicas o los certificados de defunción, como puede ser la percepción de una mujer con cáncer cervicouterino hacia el sistema de salud, o las condiciones en que alguna de ellas realizó un aborto, o las respuestas de la comunidad ante situaciones de violencia doméstica. Todos estos aspectos son dimensiones de la salud femenina que pueden permanecer velados tras la naturalización que impone la cultura y que sin embargo, aun desde la oscuridad, continúan enfermando y matando. El desafío radica entonces en lograr que emerjan los factores que inciden en la salud femenina, pero que ya sea por naturalización o negación, no son tenidos en cuenta al momento de planificar políticas de promoción de la salud.

\section{NOTA}

Este trabajo es una síntesis del proyecto de investigación doctoral, articulado en el marco del doctorado en estudios sociales agrarios del Centro de Estudios Avanzados de la Universidad Nacional de Córdoba, Argentina.

\section{FUENTES Y REFERENCIAS}

\section{Fuentes}

Datos Censo de Hogares y Población 1991 y 2001. República Argentina. Instituto Nacional de Estadísticas y Censos, Indec. Disponible en: http://www.indec.mecon.gov.ar . Acceso en: mar. 2006

Encuesta Permanente de Hogares (EPH). Disponible en http://www.indec.mecon.gov.ar. Acceso en mar. 2006.

\section{Referencias}

BECCARIA, Luis Alberto; CARCIOFI, Ricardo. Políticas públicas en la provisión y

financiamiento de los servicios sociales. In: Minujin, A. (Ed.). Desigualdad y exclusión: desafíos para la política social en la Argentina de fin de siglo. Buenos Aires: Unicef; Losada. p.193-240. 1996.

BECCARIA, Luis Alberto; LÓPEZ, Néstor (Comp.).

Sin trabajo: las características del desempleo y sus efectos en la sociedad argentina. Buenos Aires: Unicef; Losada. 1996.

BENEDETTI, Alejandro.

Susques: de 'Despoblado' a 'Pórtico de los Andes' - transformaciones territoriales en la frontera norte argentino-chilena (Siglo XX). Presentado en: Coloquio Sobre

Transformaciones Territoriales, 4: Sociedad, Territorio y Sustentabilidad: Perspectivas Desde
Informe Argentino Sobre Desarrollo Humano 1996. Senado de la Nación, en base a datos de Indec, Buenos Aires, 1996. Disponible en: http://www.desarrollohumano.org.ar/ IDHArgentina/96_arg/96_arg.html. Acceso en: oct. 2005.

el Desarrollo Regional y Local, Montevideo, Uruguay. ago. 2002.

BUSTAMANTE-MONTES, Lilia Patricia; BELLIDO-BÁRCENAS, Francisco; RIOJASRODRÍGUEZ, Horacio.

Características sociodemográficas de personas que murieron por tuberculosis pulmonar en Veracruz, México. 1993. Salud Pública de México, México D.F., v.38, n.5. p.323-331. 1996.

CAMPERO, Lourdes et al.

La contribución de la violencia a la mortalidad materna en Morelos, México. Morelos: Instituto Nacional de Salud Pública. Disponible en: http:/ /www.scielo.org. Acceso en: jul. 2006. 2006.

CÁRDENAS, Rosario.

El uso de la autopsia verbal en el análisis de la salud. Estudios Demográficos y Urbanos, México D.F., n.45, p.665-683. Disponible en: 
http://www.redalyc.uaemex.mx Acceso en: mar. 2006. sept.-dic. 2000.

CASTRO, Roberto et al.

A study on maternal mortality in México through a qualitative approach. Journal of Women's Health and Gender-Based Medicine, New Rochelle, v.9, n.6, p.679-690. 2000.

CHAM, Mamady; SUNDBY, Johanne; VANGEN, Siri. Maternal mortality in the rural Gambia, a qualitative study on access to emergency obstetric care. Reproductive Health, London, v.2, n.3. Disponible en: www.reproductive-healthjournal.com/content/2/1/3. Acceso en: mar. 2007. 2005.

CORREA, Sonia.

Salud reproductiva, género y sexualidad; legitimación y nuevas interrogantes. In: Stern, C.; Figueroa, G. (Coord.). Sexualidad y salud reproductiva: avances y retos para la investigación. México D.F.: El Colegio de México. p.127-151. 2001.

FIGUEROA, Juan Guillermo; STERN, Claudio (Coord.).

Encuentros y desencuentros en la salud reproductiva: políticas públicas, marcos normativos y actores sociales. México: El Colegio de México. 2001.

FOTTRELL, Edward et al.

Revealing the burden of maternal mortality: a probabilistic model for determining pregnancyrelated causes of death from verbal autopsies. Population Health Metrics, London, v.5, n.1. Disponible en: http: www.pophealthmetrics. com/content/5/1/1. Acceso en: jul. 2007. 2007.

FREYERMUTH ENCISO, Graciela. Methodological notes on estimating the under reporting of maternal mortality in indigenous peoples, and the qualitative study of maternal death. McArthur: s.n. 2004.

FREYERMUTH ENCISO, Graciela. Las mujeres de humo: morir en Chenalhó género, etnia y generación, factores constitutivos del riesgo durante la maternidad. México D.F.: Ciesas; Porrua. 2003.

FREYERMUTH ENCISO, Graciela.

Muerte materna, lo que no dicen las estadísticas de salud. In: Elu, María del Carmen; Santos Pruneda, Elsa (Ed.). Una nueva mirada a la mortalidad materna en México. México D.F.: Comité por una Maternidad Sin Riesgos en México. 1999.

GARRIDO, Hilda.

Mujeres y trabajo en el área de Trancas (Provincia de Tucumán). Presentado en:
CALAAR - Congreso Argentino de Antropología Rural: Antropología y Ruralidad: Un Reencuentro. Tilcara (Jujuy). mar. 2004.

GRONDA, Jorge et al.

Virus papiloma humano en mujeres quechuas jujeñas con alta frecuencia de cáncer de cuello uterino. Tipos virales y variantes de Hpv16. Revista Medicina, Buenos Aires, v.62, n.3, p.209-220. 2002.

HILL, Kennet et al.

How should we measure maternal mortality in the developing world? A comparison of household deaths and sibling history approaches. Bulletin of the World Health Organization, Washington, v.84, n.3, p.173-180. mar. 2006.

HOJ, Lars; STENSBALLE, Jakob; AABY, Peter. Maternal mortality in Guinea-Bissau: the use of verbal autopsy in a multiethnic population. International Journal of Epidemiology, London, n.28, n.1, p.70-76. 1999.

IDOYAGA MOLINA, Anatilde. Lo sagrado en las terapias de las medicinas tradicionales de Cuyo y el Noa. Revista Scripta Ethnologica, Buenos Aires, v.23, p.9-75.

Disponible en: http://www.redalyc.org. Acceso en: set. 2006. 2001.

LEÓN, Magdalena de.

El empoderamiento en la teoria y práctica del feminismo. In: Leon, Magdalena de (Comp). Poder y empoderamiento de las mujeres. Bogotá: Universidad de Colombia. 2003.

LESLIE, Joanne; RUBIN-KUTZMAN, Jane; GOLDANI, Ana María.

La definición de salud reproductiva en el contexto de la vida de las mujeres. In: Stern, C.; Figueroa, G. (Coord.). Sexualidad y salud reproductiva: avances y retos para la investigación. México: El Colegio de México. p.165-192. 2001.

LO VUOLO, Ruben; BARBEITO, Alberto. La nueva oscuridad de la política social: del Estado populista al neoconservador. Buenos Aires: Miño y Dávila. 1998.

LO VUOLO, Ruben et al.

La pobreza de la política contra la pobreza. Buenos Aires: Miño y Dávila. 1999.

LOZANO, Claudio; FELLETTI, Roberto. Las crisis provinciales. Informe Idep-ATE, Buenos Aires. 1992.

LULU, Kidest; BERHANE, Yemane. The use of simplified verbal autopsy in identifying causes of adult death in a predominantly rural population in Ethiopia. BMC Public Health, London, v.5, n.58. 
Disponible en: http://www.biomedcentral.com/ 1471-2458/5/58. Acceso en: mar. 2007. 2005.

MENÉNDEZ, Eduardo.

Modelo hegemónico, modelo alternativo subordinado, modelo de auto atención. Caracteres estructurales. In: Campos, R.

(Comp.). La antropología médica en México. t.1. México: Instituto Mora-Universidad Autónoma Mora. p.97-114. 1992.

MINUJIN, Alberto.

En la rodada. In: Minujin, Alberto; Beccaria, Luis Alberto, Bustelo, L. (Ed.). Cuesta abajo: los nuevos pobres - efectos de la crisis en la sociedad argentina. Buenos Aires: Unicef; Losada. 1992.

OSZLAK, Oscar.

De menor a mejor: el desafío de la 'segunda' reforma del Estado. Revista Nueva Sociedad, Caracas, n.160. mar.-abr.1999.

RAMOS, Silvina et al.

Para que cada muerte materna importe. Buenos Aires: Ministerio de Salud de la República Argentina y Cedes. Disponible en: http://www.cedes.org.ar. Acceso en: nov. 2006. 2004.

ROITTER, Mario.

El tercer sector como representación topográfica de sociedad civil. In: Mato, Daniel (Coord.). Políticas de ciudadanía y sociedad civil en tiempos de globalización. Caracas: Faces; Universidad Central de Venezuela. 2004.

ROMERO, Mariana; RAMOS, Silvina. Estudio colaborativo multicéntrico: mortalidad materna en la Argentina - diagnóstico para la reorientación de políticas y programas. Buenos Aires: Cedes. Disponible en: http:// www.cedes.org.ar. Acceso en: nov. 2006. 2004.
RONSMANS, Carine et al.

A comparison of three verbal autopsy methods to ascertain levels and causes of maternal deaths in Matlab, Bangladesh. International Journal of Epidemiology, London, v.27, n.4, p.660-666. 1998.

SALGADO MARTíNEZ, Carolina.

Cuatro líneas de reflexión en torno a los elementos sociales de la salud reproductiva (Comentario). In: Stern, C.; Figueroa, G. (Coord.). Sexualidad y salud reproductiva: avances y retos para la investigación. México D.F.: El Colegio de México. p.115-126. 2001.

SALLES, Vania; TUIRÁN, Rodolfo.

El discurso de la salud reproductiva: ¿Un nuevo dogma? In: Stern, C.; Figueroa, G. (Coord.). Sexualidad y salud reproductiva: avances y retos para la investigación. México D.F.: El Colegio de México. p.93-112. 2001.

SETEL, Philip et al.

Core verbal autopsy procedures with comparative validation results from two countries. Plos Medicine, San Francisco, v.3, n.8, p.1282-1291. Disponible en: http://

www.plosmedicine.org. Acceso en: mar. 2007. 2006.

SETEL, Philip et al.

Sample registration of vital events with verbal autopsy: a renewed commitment to measuring and monitoring vital statistics. Bulletin of the World Health Organization, Washington, v.83, n.8, p.561-640. Disponible en: http:// www.who.int. Acceso en: mar. 2007. ago. 2005.

SLOAN, Nancy et al.

The etiology of maternal mortality in developing countries: What do verbal autopsies tell us? Bulletin of the World Health Organization, Washington, n.79. p.805-810. 2001. 